\title{
Iakovos Pylarinos (1659-1718) und sein Beitrag zur Variolation
}

\author{
Von Stephanos Geroulanos
}

Die Herkunft der Pockeninokulation oder Variolation ist unbekannt. Ihr Ursprung reicht in sehr alte Zeiten zurück und ist mit größter Wahrscheinlichkeit eine rein empirische Erfindung. Ihre Anwendung war vielleicht schon in den ersten Jahrhunderten der christlichen Zeitrechnung in Rom ${ }^{1}$ und wahrscheinlich um das Jahr 590 n Chr. in China ${ }^{1,2}$ bekannt. Das Inokulationsverfahren, wie es um 1670 in Konstantinopel eingeführt wurde, war laut E.Timonis $(1714)^{3}$ um 1650 in Zirkassien, Georgien und im Kaukasus mit Sicherheit bekannt ${ }^{4}$. Nach I. Pylarinos $(1715)^{5}$ kannte man im 17. Jahrhundert die Pockeninokulation auch in Griechenland. Er selber hat die Methode 1701 von einer Griechin aus Thessalien gelernt. Übrigens soll 1716 das gleiche Verfahren auch unter den schwarzen Sklaven in Amerika bekannt gewesen sein ${ }^{6}$; ebenso war es am Anfang des 18. Jahrhunderts in Nordafrika ein vielgeübter Brauch ${ }^{7}$.

In Europa wurde die Inokulation durch Emmanuel Timonis und Iakovos Pylarinos eingeführt und jahrzehntelang als byzantinische ${ }^{7}$ und später als griechische ${ }^{8}$ Methode bezeichnet. Iakovos Pylarinos scheint der erste uns bekannte Arzt gewesen zu sein, der die Methode 1701 bei den Kindern eines Freundes angewendet hat. Er berichtete über seine Methode 1715, nachdem er bereits mehrere hundert Personen geimpft und über längere Zeit beobachtet hatte ${ }^{5}$. Emmanuel Timonis, der $1714^{3}$, also ein Jahr vor Pylarinos, darüber schrieb, berichtete über 50 inokulierte Patienten der letzten acht Jahre, ohne zu erwähnen, ob er sie selber geimpft habe. Lady Mary Wortley Montagu, die Frau des englischen Botschafters in Konstantinopel, die 1721 die Methode in England lancierte ${ }^{1,8,9}$, schrieb ihren ersten Brief der berühmten Serie aus Adrianopel an ihre Freundin Miss Sarah Chiswell am 1. April 1717². Sie berichtet, daß eine Menge alter Frauen diese Operation im September ausübe und daß sich alljährlich Tausende dieser Operation unterziehen. Ein tödlicher Verlauf sei niemals beobachtet worden: «und glaube mir, ich bin von der Sicherheit dieses Experimentes so vollkommen befriedigt, daß ich die Absicht habe, dasselbe an meinem lieben kleinen Sohn zu versuchen.» Kurz danach soll Timonis denn auch ihren Sohn inokuliert haben ${ }^{9}$.

Über E.Timonis ist bereits viel geschrieben worden. Der Beitrag von Pylarinos ist jedoch mit wenigen Ausnahmen unbeachtet geblieben. Wir möchten deswegen darauf eingehen. 


\section{Biographie}

Iakovos Pylarinos ${ }^{10}$, Sohn des Dimitrios, wurde 1659 in Lixourion auf der Insel Kephalonia geboren (nach Mazarakis ${ }^{11}$ am 9. Januar, nach Pignat ${ }^{12}$ am 9. Juni).

Dem Wunsche seiner Eltern folgend, studierte er zuerst klassische Philologie und Rechte an der Universität Padua. Mit 16 Jahren erhielt er sein Diplom für die Rechte (Laureat) und kehrte nach Kephalonia zurück, wo er während kurzer Zeit als Rechtsanwalt praktizierte ${ }^{13}$. Doch bald fuhr er nach Padua zurück und erwarb mit 20 Jahren den Doktortitel der Medizin. Als wahrer Landsmann des Odysseus ${ }^{14}$ hat er die damals bekannten Erdteile erkundet. Kurz nach seinem Studium finden wir ihn in Kreta, wo der damalige Gouverneur der Insel, Ismail Pascha, ihn zum Hofarzt ernannte (1680). Etwas später, nach kurzem Aufenthalt in Konstantinopel, wurde er in Moldovalachien (Rumänien) Archiatros (Chefarzt) des Gouverneurs der Region, des Prinzen Cantacuzènos. Drei Jahre später, nach einem kurzen Aufenthalt in seiner Heimat (wo sein Vater starb) besuchte er Deutschland und Rußland. In der neuen Hauptstadt des russischen Kaiserreiches ernannte ihn Peter der Große zum Leibarzt. Seine schlechte Gesundheit und die nördliche Kälte zwangen ihn jedoch, den russischen Hof zu verlassen und nach Kephalonia zurückzukehren. Zwischendurch soll er Ägypten und Halepion im östlichen Mittelmeer besucht haben, möglicherweise als Morozinis Flottenarzt ${ }^{12}$. Um 1700 finden wir ihn in Serbien als Leibarzt des serbischen Herrschers mit einem jährlichen Honorar von 1500 Florin $^{15}$. Von hier aus reiste er nach Venedig und Padua.

1701 ließ er sich in Konstantinopel nieder, wo im gleichen Jahr eine schwere Pockenepidemie ausbrach. Ein Freund, der Kinder hatte und deren Ansteckung befürchtete, machte ihn mit einer Griechin aus Thessalien bekannt, die angeblich schon Tausende von Personen geimpft hatte. Pylarinos ließ sich nicht vom mysteriösen und theurgischen Ruf der Impfung beeinflussen und erkannte sofort die wesentlichen Punkte. Er impfte die drei Kinder seines Freunde am Oberarm ${ }^{15}$. Wie er später schreibt, zeigten die zwei jüngeren Kinder (die 7 und 8 Jahre alt waren) nur eine leichte Pustelbildung und waren nur während einer Woche leicht krank. Der 18jährige Sohn wurde viel schwerer krank und zeigte während 14 Tagen hohes Fieber. Pylarinos erklärte diese unterschiedlichen Reaktionen als Idiosynkrasie. In der Folge übte Pylarinos die Impfung an zahlreichen noblen Familien in Konstantinopel aus.

1712 lebte er in Smyrna als Konsul der venezianischen Republik. Nach Alivisatos $^{15}$ soll er hier um 1712 seinen Freund und Schulkameraden aus Padua, Emmanuel Timonis, der aus Chios stammte, wieder getroffen und ihn über die Variolation unterrichtet haben. 
1714 kehrte Pylarinos nach Italien zurück, um seine Kollegen über seine Krankheit, den Morbus hydropicus, zu konsultieren. Er ließ in Venedig im Februar 1715 sein Buch über die Variolation lateinisch drucken ${ }^{13}$. Es erschien in Venedig und Padua am 10. November 1715 und wurde erneut 1717 in Nürnberg und 1718 in Leyden aufgelegt ${ }^{12}$. Eine Kurzfassung erschien in den «Philosophical Transactions » von 1716 (wahrscheinlich eingeschickt durch William Sherard, den englischen Konsul in Smyrna, dem das Buch gewidmet war ${ }^{16}$ ). In der Folge veröffentlichte Pylarinos weitere Bücher. Das wichtigste war «La medicina diffesa », gedruckt 1717 in Venedig als Antwort auf das Buch: «Die von den lügenden Ärzten betrogene Welt» des Arztes und Mathematikers Josef Gazola ${ }^{12}$.

Pylarinos starb am 17. Juni 1718 in Padua ${ }^{12}$. Er wurde in der Kirche von San Francesco in Padua begraben, wo sein Grab immer noch zu sehen ist. Auf dem Grabmal steht auf Griechisch, daß er in Italien, Rußland, der Türkei und in Ägypten berühmt war ${ }^{17,18}$. J.-P. Niceron ${ }^{19}$ zählt ihn in seinem Buch «Mémoires pour servir à l'histoire des hommes illustres dans la république des lettres» (1729-1745) zu den berühmtesten Ärzten seiner Zeit. Zeno ${ }^{11}$ soll seine Biographie geschrieben haben, und Morelli ${ }^{13}$ nennt ihn «Uomo di vasto genio e di talento perspicacissimo».

\section{Die Inokulationsmethode}

Die Pockeninokulation beruht nach Pylarinos in der Einführung (insertio) von Pockenserum (fermentum morbificum) durch kleine Skarifikationen in die Haut. Das Pockenserum wird aus Pockenpusteln entnommen. Pylarinos schildert seine Technik in folgender Weise ${ }^{5}$ :

«Als beste Zeit für die Impfung gilt der Frühling; der Winter soll ausgeschlossen werden... Den Eiter für die Transplantation muß man mit größter Vorsicht sammeln. Das Serum soll im Laufe einer Epidemie aus einer reifen Pustel entnommen werden. Der Patient, dem der Eiter entnommen wird, soll jung sein und einen guten Allgemeinzustand aufweisen; er soll die Pocken leicht überstanden haben ... Der Eiter soll in einem sauberen Glas gesammelt werden und darf nicht abkühlen. Beim Transport soll das Glas innerhalb der Kleider in der Achselhöhle aufbewahrt werden, so daß es ständig die Körpertemperatur behält ... Die Transplantation muß möglichst rasch durchgeführt werden; der Impf kandidat soll in einem warmen Zimmer sein ...

Durch ein scharfes Instrument aus Metall oder Gold, das waagrecht gehalten wird, werden einzelne kleine Inzisionen gemacht, mit Vorteil am Arm oder Oberschenkel oder an der Haar-Stirngrenze. Der Eiter wird anschließend mit dem gleichen Instrument tropfenweise auf die Einschnitte gegeben, und die Wunden werden vorsichtig gedeckt. Der Geimpfte darf die Transplantationsstellen nicht berühren und muß vermeiden, daß die Stellen naß werden ... Wählt man Regionen über muskelkräftigen Gebieten, so können starke lokale Entzündungsreaktionen vermieden werden, und die Sehnenregionen 
bleiben unberührt ... Der Geimpfte muß mehrere Tage zu Hause im Bett bleiben und eine strenge Diät von mindestens 40 Tagen einhalten ... Die Symptome unterscheiden sich je nach Alter, Idiosynkrasie und Allgemeinzustand des Patienten ... Ebenso kann das Pustelserum (fermentum) in seiner Stärke verschieden sein, so daß gelegentlich die ersten Pusteln schon am 3. oder 4. Tag oder aber erst sehr viel später auftreten ... Im allgemeinen erscheinen jedoch die ersten Pusteln am 7.Tag. Sie liegen weit auseinander; es sind meist 20-30, ganz selten 100 oder 200 an der Zahl. Am allerersten erscheinen sie an der Stelle der Skarifikation. Sie beginnen mit einem leichten örtlichen Erythem, dem mit der Zeit eine kleine Phlyktäne folgt. Ganz selten treten eitrige Ulzerationen auf, die sehr schmerzhaft sind, vor allem, wenn sie an den Händen oder Füßen lokalisiert sind. Sobald sie aber sich öffnen, verschwinden alle Symptome.»

Pylarinos hat trotz der großen Anzahl der Pockeninokulationen nie einen tödlichen Fall gesehen. Er empfiehlt aber, daß die Inokulation von erfahrenen Ärzten durchgeführt werden müsse. Sollten keine Reaktionen auftreten, so beruhe dies auf der Idiosynkrasie des Patienten, auf einer früher unbemerkt abgelaufenen Pockeninfektion oder auf der Inaktivität des Pustelserums. Er empfiehlt dann die Wiederholung der Inokulation.

\section{Diskussion}

Im allgemeinen nimmt man an, daß die Pocken im Altertum unbekannt waren und daß sie später aus Afrika, Indien oder China zu uns kamen. Galen erwähnt die Pocken nicht. Als Erstbeschreiber gilt der Araber Rhazes, der sie um 910 n. Chr schilderte ${ }^{8}$, aber als weniger gefährlich als die Masern betrachtete. Oidtmann ${ }^{20}$ (1880) meint dagegen, daß die Pocken schon viel früher bekannt waren. Er schreibt:

«Einer der ältesten Schriftsteller über die Pocken der Menschen ist ein Priester namens Aaron aus Ägypten, welcher im Jahre 622 z. Z. Mohameds in Alexandrien lebte (Abul Pharaz. p. 99). Von ihm existiert in syrischer Sprache die Beschreibung einer Pockenepidemie, welche aus Ägypten nach Arabien verschleppt worden war. In Griechenland waren nach Paulus Aeginae im Jahre 641 die Pocken noch unbekannt. Ein jüdischer Arzt, der weise Maserjawaihus aus Basor, welcher die medizinischen «Pandekten» des ägyptischen Aaron aus den Schriften ins Arabische übersetzt hat, beschreibt im Jahre 683 als Folge der Pocken Augenentzündungen und Erblindung (Rhazes Contin, 421). Im Jahre 753 starb ein Chalif an den Pocken (Rhazes 419). Anno 794 lehrte in Bagdad der berühmte Johannes, Sohn Mesues, die Pocken und Pockennarben mit Wundertränken zu behandeln. Mesue und Isaak, Urenkel des Johannes von Bagdad, verordneten im 9. Jahrhundert als unentbehrliches Schutz- und Heilmittel gegen die Pocken Schröpfköpfe und Aderlaß.»

Sollten diese Angaben stimmen, so wären die Pocken schon einige Jahrhunderte vorher in Kleinasien, im mittleren Osten und in Ägypten beheimatet und gut 
bekannt gewesen. Beim regen Handelsverkehr müßten sie auch in andere Städte in Griechenland und Europa verschleppt worden sein; doch kann dies nicht mit Sicherheit gesagt werden. Von besonderer Bedeutung scheint mir in diesem Zusammenhang die Übersetzung von Oidtmann ${ }^{20}$ aus Vergils Georgica zu sein, bei dem unter Abschnitt I, 2 folgendes steht: «Das Pockengift kann aus den Schafskadavern weder durch Wasser noch durch Hitze entfernt werden. Niemand darf die von den Pocken angefressenen Felle scheren, noch die faulschweißigen Stoffe aus solcher Schafswolle betasten, sonst wird er von brennenden Blattern befallen, und schmutziger Schweiß bricht aus seinen Gliedern hervor.» Es scheint also, daß die Schafspocken zu Vergils Zeiten in Rom gut bekannt waren. Wenn es nun in Kleinasien Pockenepidemien gab und wenn in Rom die Schafspocken bekannt waren, so fragt man sich, warum die Pocken von den großen Ärzten wie Galen oder Paulus unbemerkt geblieben sind. Hier kann vielleicht eine Vermutung weiterhelfen.

Da das Pockenvirus gegen Eintrocknung resistent ist, war wahrscheinlich damals der ganze Kleider- und Bettenbestand der Bevölkerung, der aus Schafswolle stammte, mit Schafspocken infiziert. Jedes Kind wurde somit frühzeitig mit Schafspocken angesteckt, wahrscheinlich sogar schon zur Zeit, wo es noch mütterliche Abwehrstoffe besaß. Die Erkrankung verlief daher sehr mild und wurde möglicherweise von den damaligen Ärzten als harmlose Kinderkrankheit angesehen, weswegen Rhazes die Pocken für harmloser als die Masern hielt. Der enge Kontakt der vorwiegend landwirtschaftlichen Bevölkerung mit den Schafen half weiterhin mit, die Immunität lebenslang aufrechtzuerhalten. Als später die Bevölkerung aus Sicherheitsgründen in die Städte zog, entfernte sie sich von ihren natürlichen frühkindlichen Infektionsquellen. Ihre natürliche Immunität verschwand, und die Pocken konnten Fuß fassen.

Diese Kreuzimmunität mit den Schafspocken wurde später (1806) durch Sacco, den Direktor des Impfwesens in Italien, ausgenützt. Er impfte Kinder mit Lymphe aus den Pusteln pockenkranker Schafe (Ovination) und erzielte eine gleiche Immunität für echte Pocken wie Jenner 1978 mit der Vakzination ${ }^{20}$.

Ebenso wie die Pocken möglicherweise viel verbreiteter waren, als wir bisher meinten, sind vielleicht verschiedene Pockenverhütungsmethoden viel früher und häufiger praktiziert worden, als es im allgemeinen angenommen wird. Schon 590 n. Chr. soll in China ein Verhütungsverfahren mit Erfolg ausgeübt worden $\operatorname{sein}^{1,2}$. Aber auch in Europa waren schon frühzeitig Methoden bekannt, so das Pockenkaufen. Von der Voraussetzung ausgehend, jedem Menschen sei das Pockengift angeboren und müsse sich in einem Blatternausbruch ausschäumen, und im Wahn befangen, daß ein Mensch, der nicht geblattert habe, das 50. Lebens- 
jahr nicht erreiche, gaben sich die Leute alle Mühe, sich und ihre Kinder mit dem Gift pockenkranker Menschen zu infizieren. So war es Sitte, daß man sich unter eigentümlichen Zeremonien die Hemden pockenkranker Kinder lieh und sie den eigenen Kindern anzog, damit diese auch die Pocken bekämen. Der Pfarrer Cuno von Salzwedel war einer der ersten, der zu Beginn des 16. Jahrhunderts diese Methode der Weiterverpflanzung der Pocken beschrieb ${ }^{20}$. Den pockenkranken Kindern wurden für das Leihen der schmutzigen Wäsche einige Geldstücke, meist nur Pfennige, bezahlt; daher stammt der Ausdruck «Pockenkauf». Eine andere Art, absichtlich Pocken hervorzurufen, war die, daß gesunde Kinder zu pockenkranken in das Bett gelegt wurden. Erwachsene nahmen Krusten von Pockenpusteln in die Hand, um sich anzustecken. Diese Verfahren waren in der Bevölkerung verbreitet und wurden zum Teil von der Medizin, zum Teil von der Kirche sehr bekämpft.

Ebenso wurde die Pockeninokulation weithin ausgeübt. Zur Zeit von Timonis und Pylarinos zu Beginn des 18. Jahrhunderts geschah dies im Peloponnes ${ }^{21}$, in Thessalien $^{5}$, Italien ${ }^{22}$, in der Türkei ${ }^{21}$, in Armenien ${ }^{22}$, Georgien $^{3}$, Zirkassien ${ }^{3}$, Persien $^{21}$, in der arabischen Wüste ${ }^{23}$, in Indien $(1767)^{7}$ und in Nordafrika ${ }^{7}$, ebenso bei den Sklaven von Nordamerika ${ }^{6}$. Die Methode wurde jedoch von nichtärztlichen Personen durchgeführt und von den Ärzten des Mittelalters bekämpft. Man muß sich deshalb nicht wundern, daß die Methode, als sie endlich von den Ärzten übernommen wurde, rasch Verbreitung fand. 1768, also knapp 50 Jahre nach den Veröffentlichungen von Pylarinos und Timonis, berichtet Krünitz in seiner Literaturübersicht, daß bereits 317 Schriftsteller über das Impfen geschrieben hätten ${ }^{24}$. Wie groß der Wert dieses Vorgehens war, zeigt die Arbeit von Stark $^{9}$, oder berichtet, wie Washington 1777 seine gesamte Armee zwangsweise inokuliert und daher den Befreiungskrieg über die Engländer gewonnen habe.

Von besonderer Bedeutung sind die statistischen Angaben von Stark (1977). Obwohl man mitten in Epidemien impfte und einige der Patienten zur Zeit der Impfung schon infiziert waren, und obwohl wahllos Kinder, Erwachsene, Alte und Kranke inokuliert wurden, schwankte die Letalität in Boston nur zwischen $3 \%$ (1721) und 1,5\% (1752). Die gleichzeitige Mortalität der nicht geimpften Bevölkerung, die zum Teil durch frühere Epidemien immun war, lag bei $12,5 \%$ bzw. 9,1\%. Unter 1000 von Kirkpatrick inokulierten Personen ${ }^{25}$ in Charleston, South Carolina, lag sie sogar bei $0,8 \%$. Von 2000 in Südengland Geimpften sollen nur zwei schwangere Frauen gestorben sein $^{25}$. Eine so geringe Letalität hätte sich auch Jenner gewünscht.

Wenngleich die Inokulation schon so verbreitet war, ist es das große Verdienst von Pylarinos, die Pockeninokulation aus der Empirie herausgeholt und in die 
wissenschaftliche Medizin eingeführt zu haben. Er erkannte sofort, daß nicht das Theurgische den Schutz verlieh, sondern die «Transplantation der Mater», die Pustelflüssigkeit. Er war der erste, der die lebenslange Immunität nach der Inokulation hervorhob. Pylarinos stellte fest, daß es viel besser sei, über muskelkräftigen Stellen am Oberarm oder am Oberschenkel statt im Gesicht oder an den Händen zu impfen, um Narben und Tendovaginitiden zu vermeiden. Vor der Bekanntgabe seiner Inokulationsmethode wartete er den Erfolg seiner Impfungen bei den nächsten Epidemien ab. Erst nachdem er Hunderte bis Tausende (casus innumeros) von Personen geimpft und sie über mehrere Jahre beobachtet hatte, veröffentlichte er sein Verfahren, im Gegensatz zu E.Timonis, der über 50 Fälle berichtete, die möglicherweise nicht einmal durch ihn selbst geimpft worden waren. Timonis schreibt nämlich: «It is now 8 years since $I$ have been an Eye-witness of these operations. » ${ }^{3,26}$

Pylarinos hatte das Unglück, erst als zweiter die Inokulationstechnik zu veröffentlichen. Sein Landsmann Timonis, der zur gleichen Zeit wie Pylarinos in Konstantinopel und Smyrna wirkte und möglicherweise sein Freund und Studienkollege aus Padua war, kam ihm zuvor. Lady Montagus Verdienst besteht darin, daß sie die britische Gesellschaft vom Nutzen des Impfens überzeugte ${ }^{22,28}$. Die wissenschaftlichen Grundlagen jedoch stammen von Pylarinos und Timonis, die einige Jahre zuvor in den «Philosophical Transactions» ihre Erfahrungen bekanntgaben $^{3,5}$. Die weltweite Anwendung der Methode, vor allem in Amerika und Europa, verdanken wir dem Einfluß der Royal Society und ihrer Zeitschrift, der «Philosophical Transactions».

Pylarinos und Timonis waren die großen Vorgänger von Edward Jenner (1749-1823), der dann statt des Pockenserums 1798 das Kuhpockenserum empfahl ${ }^{27}$.

\section{Zeittafel}

Die wichtigsten Daten zur Geschichte der Variolation (nach Alivisatos, Cavadias, Kübler, Miller und Stark):

Ca. 590 n. Chr.: Pockenprophylaxe durch Einatmung von Pockenkrusten in China ${ }^{1,2}$.

Ca. 1600: Variolation in Zirkassien und Georgien ${ }^{3}$; $(?)^{29}$.

Vor 1670: Einführung in Griechenland (Thessalien) ${ }^{5}$; erste Inokulation in Europa.

Ca. 1670: Einführung in Konstantinopel ${ }^{3,5}$.

1701: Erste bekannte Anwendung der Impfung durch einen Mediziner (I. Pylarinos) ${ }^{5}$.

1714: Der vom Dezember 1713 datierte Brief von E. Timonis wird im Frühling 1714 vor der Royal Society in London von Prof. John Woodward vorgelesen und in den Philosophical Transactions vom Mai/Juni 1714 gedruckt $^{3}$. 
1715: Die Monographie von I. Pylarinos über die Inokulationsmethode erscheint in Vene$\operatorname{dig}^{5}$. Erste Inokulationen durch Pylarinos in Padua und Venedig.

Peter Kennedy aus Schottland teilt in seinem Reisebericht mit, daß auf dem Peloponnes, in der Türkei und in Persien die Inokulationsmethode weite Anwendung findet ${ }^{21}$.

1716: Der wesentliche Anteil der Monographie von Pylarinos wird im Januar der Royal Society durch Sir Hans Sloane vorgetragen und in den Philosophical Transactions vom Januar/Februar/März 1716 wieder gedruckt ${ }^{5}$.

1717: Am 1. April wird Lady Mary Wortley Montagus berühmter Brief aus Adrianopel an Miss Sarah Chiswell abgeschickt ${ }^{1,30}$.

Die Monographie von Pylarinos wird in Nürnberg übersetzt und gedruckt ${ }^{12}$.

Lady Mary Wortley Montagu trifft Timonis in Konstantinopel ${ }^{1,9}$.

1718: Die Monographie von Pylarinos wird in Leyden wiederaufgelegt ${ }^{13}$.

1720: Ulrich und Stoll schreiben ihre Doktorarbeit über die Variolation in Wittenberg ${ }^{31}$.

1721: Lady Mary impft ihre Tochter in London. Erstimpfung in England.

Sechs zum Tode verurteilte Kriminelle erhalten ihre Freiheit, nachdem sie sich in London zu Versuchszwecken der Variolation unterzogen haben ${ }^{8}$.

Dr. Zabdiel Boylston führt die erste Variolation in Boston durch ${ }^{9}$.

1722: A. Le Duc führt die Variolation in Holland ein ${ }^{\text {? }}$.

1723: Erste Inokulation in Frankreich durch de la Costa ${ }^{1}$.

1728: 897 Inokulationen in England mit 17 Todesfällen $(1,9 \%)^{9}$.

1738: Kirkpatrick inokuliert in South Carolina ca. 1000 Personen mit nur 8 Todesfällen $(=0,8 \%)$. Bei der gleichzeitigen Epidemie sterben $12,5 \%$ der Bevölkerung South Carolinas ${ }^{25}$.

Erste Abteilung für Variolation in einem neugebauten öffentlichen Spital in London ${ }^{13}$.

1748: Théodore Tronchin aus Genf verbreitet die Variolation in Holland ${ }^{32}$.

1750: Inokulation der Kinder des Herzogs von Orléans in Paris ${ }^{15}$.

Der Chirurg Daniel Guyot pfropft in Genf der 18jährigen Frau Gallatin-Tronchin die Pocken ein ${ }^{32}$.

1753: Erste Inokulation in Lausanne ${ }^{32}$.

1754: Das «Royal College of Physicians» erklärt die Inokulation als «highly salutary » ${ }^{\mathbf{2}}$.

1756: Erste Inokulation in Basel durch Achilles Mieg ${ }^{32}$.

1766: Daniel Sutton impft innert zweier Jahre in England 20000 Personen mit nur wenig Todesfällen ${ }^{1}$.

1777: George Washington impft seine ganze Armee und schreibt an Gouverneur Trumbull: «inoculation ... has been attended by amazing success ${ }^{9}$.

1779: Abteilung für Variolation im Kaiserlichen Spital von Irkutsk (Sibirien) ${ }^{13}$.

1798: Veröffentlichung der Methode der Vakzination durch Edward Jenner ${ }^{28,33}$.

1806: Inokulation von Kindern durch Dr. Sacco mit Pockenlymphe aus Schafen (Ovination). Die Methode findet keine breitere Anwendung, da die Vaccinia leichter zu gewinnen ist ${ }^{9}$.

Ca. 1850: Verbot der Inokulation in England ${ }^{34}$. 


\section{Anmerkungen}

1 Kübler, P., Geschichte der Pocken und der Impfung, Hirschwald, Berlin 1901.

2 Die chinesische Pockeninokulationsmethode unterscheidet sich von der später in Europa gebräuchlichen, indem Blasenkrusten, mit Moschus vermischt, mit einem Baumwollbäuschchen in die Nase eingeführt wurden, nachdem sie zur Milderung der Wirkung jahrelang auf bewahrt und mit Dämpfen von Heilkräutern durchräuchert worden waren.

3 Timonis, Emmanuel, An Account, or History of the Procuring the Small Pox by incision or inoculation; as it has been for some time been practised at Constantinople; being the Extract of a letter dated at Constantinople, December 1713. In: Philosophical Transactions 339 (1714), S.72-82.

4 In Zirkassien, Georgien und im Kaukasus inokulierte man die kleinen Mädchen sehr frühzeitig, um sie später zu einem höheren Preis verkaufen zu können ${ }^{3}$.

5 Pylarinus, Jacobus, Nova et tuta Variola excitandi per Transplantationem Methodus, nuper inventa et in usum tracta: qua ritè peracta, immunia in posterum praeservantur ab hujusmodi contagio Corpora, Venetiis MDCCXV (1715) apud Jo. Gabrielem Hertz. Kurzfassung in: Philosophical Transactions 339 (1716), S. 393-399.

${ }^{6}$ In einem Brief von Cotton Mather (datiert 16.12.1716), der die Arbeiten von Timonis und Pylarinos in den Philosophical Transactions gelesen hatte, an Dr. John Woodward von der Royal Society steht folgendes: «enquiring of my Negroman Anesimus (a native of Fezzan in South Tripolis) ${ }^{22}$, who is a pretty intelligent fellow, wether he ever had ye small pox; he answered, both yes and no; and then told me that he had undergone an operation, which had given him something of ye small pox, and would forever preserve him from it.» Zitiert nach R. B. Stark, Immunization saves Washington's Army. In: Surgery, Gynecology and Obstetrics 144 (1977), S. 425-431.

7 Le Duc, De Byzantine variolarum insitione, Leyden 1722. Es ist merkwürdig, daß über 250 Jahre nach dem Fall von Konstantinopel (1453) und der Auflösung des byzantinischen Reiches das Verfahren der Pockeninokulation als byzantinische Pfropfung bezeichnet wird. Es ist möglich, daß es diesen Namen bekam, weil es durch Byzantiner von Konstantinopel nach Europa gelangte. Es ist aber nicht ausgeschlossen, daß die Methode schon früher geübt wurde und den Byzantinern bekannt war. Dafür spricht ihre bereits damals weltweite Ausbreitung und die kreuzweise Durchführung im Sinne der Wundmale Christi, die die Methode in Zusammenhang mit der christlichen Religion brachte. Timonis berichtete 1714, daß die Methode 50 Jahre zuvor von Zirkassien, vom Kaukasus und von Georgien her nach Konstantinopel kam. Pylarinos fand sie 1715 in Thessalien. Peter Kennedy ${ }^{22}$, ein schottischer Arzt, beschrieb sie 1715 als ein im Peloponnes, in der Türkei und in Persien weitverbreitetes Verfahren. Cotton Mather ${ }^{6}$ fand es 1716 bei den amerikanischen Negern, die die Methode aus Afrika mitbrachten. Nach dem Zeugnis des damaligen arabischen Gesandten in London, Cassem Agha ${ }^{1}$, soll die Inokulation in Tripolis, Tunis und Algerien zu Anfang des 18. Jahrhunderts ein vielgeübter, aus undenklicher Zeit überlieferter Brauch gewesen sein. Holwell traf 1767 die Methode in Indien an ${ }^{1}$. Viele dieser Gebiete waren alte byzantinische Provinzen oder angrenzende Länder. Interessant ist auch die Beobachtung von Pylarinos, daß die türkischen Herrscher mohammedanischen Glaubens der Methode sehr mißtrauten; möglicherweise entsprang ihre Abneigung der kreuzartigen Weise der Durchführung unter gleichzeitigen christlichen Gebeten. 
${ }^{8}$ Ackerknecht, Erwin H., Geschichte und Geographie der wichtigsten Krankheiten, F. Enke Verlag, Stuttgart 1963, S. 55.

9 Stark, R.B., Immunization saves Washington's army. In: Surgery, Gynecology and Obstetrics 144 (1977), S. 425-431.

10 Der Name Pylarinos bedeutet «der aus Pylaros Stammende». Pylaros wird eine Gegend der Insel Kephalonia genannt, aus der die Familie von Pylarinos ursprünglich stammen sollte. Sitz der Familie soll aber bereits damals seit langem die Stadt Lixourion gewesen sein, in der immer noch Namensgenossen und möglicherweise Nachkommen leben. Jacobus Pylarinus ${ }^{5}$ ist die lateinische Übersetzung des griechischen Namens.

11 Mazarakis, A., Bibliographie berühmter Männer aus Kephalonien, Venedig 1843, S.119 (griechisch).

12 Tsitselis, Elias, Kephaliniaka Symmicta, Athen 1905 (griechisch).

13 Alivisatos, C. N., The first immunologist, James Pylarino (1659-1718), and the Introduction of Variolation. In: Proceedings of the Royal Society of Medicine 27 (1934), S.1099 bis 1104 .

14 Kephalonia gehörte zusammen mit Zakynthos (Zante) und Ithaka zum Königreich des Odysseus. Aus diesen beiden Inseln holte Odysseus den Teer, mit dem er die Kiele seiner Schiffe überstrich, die darum von Homer als schwarzkielig bezeichnet wurden (Bedfort, E., Reisen mit Odysseus, DTV-Dokumente 1971).

15 Alivisatos, C. N., Les initiateurs de la variolation au cours du XVII ${ }^{\text {ème }}$ siècle. In: Gazette médicale de France 1934, S. 628-637.

16 Illustrissimo, Praeclarissimo atque Eruditissimo Viro Wilhelmo Serhad, Dignissimo pro Inclyta Natione Britannica nunc Smyrnis Consuli.

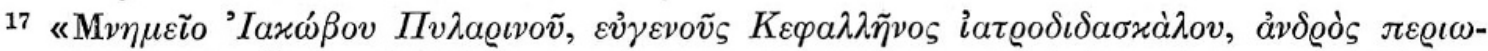

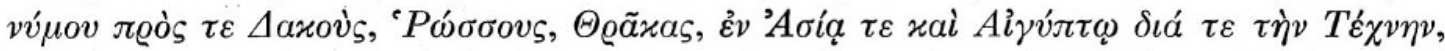

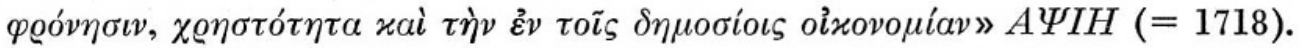

18 Denkmal des Medizinallehrers und Edelmannes aus Kephalonia, Iakovos Pylarinos, der unter den Rumänen, Russen, Thrakern, in Asien und Ägypten für seine Kunst, Einsicht und Klugheit, Ehrenhaftigkeit und Rechtschaffenheit sowie für seinen Sinn für Staatswirtschaft berühmt war. 1718. (Nach Harokopos, S., und Harokopos, Ph., Eptanissiaki Iatriki, hrsg. vom griechischen Gesundheitsministerium, Athen 1976, S.108.)

19 Niceron, J.-P., Mémoires pour servir à l'histoire des hommes illustres dans la république des lettres, 43 Bände, Paris 1729-1745.

20 Oidtmann, Heinrich, Geschichte der Pocken; ein Kulturkampf der Medicin, Herder-Verlag, Frankfurt 1881.

${ }^{21}$ Kennedy, Peter, An Essay on external Remedies (ingrafting the small pox), London 1715.

22 Miller, Geneviève, The Adoption of Inoculation for Small Pox in England and France, Philadelphia Univ. Press 1957.

23 Tarry, E., Letter to Sir Hans Sloane, August 2, 1721. Sloane MSS $4061 \mathrm{f} 164$. Die Information wurde Tarry vom griechischen Patriarchen von Antiochien gegeben.

${ }^{24}$ Krünitz, J. G., Verzeichniss der vornehmsten Schriften von den Kinderpocken und deren Einpfropfung, Leipzig 1768. Zitiert nach Kübler und Oidtmann. Siehe Koelbing, H.M., Im Kampf gegen Pocken, Tollwut, Syphilis, Reihe Gute Schriften, Basel 1974.

${ }^{25}$ Kirkpatrick, J., Erläuterung der Einpfropfung, welche die Geschichte, Theorie und Ausübung derselben nebst bei Gelegenheit gemachten Beobachtungen von den merkwürdigsten 
Erscheinungen der Kinderblattern in sich begreift. Aus dem Englischen übersetzt von Heineken, Zelle und Leipzig 1756.

${ }^{26}$ Timonis, E., a. a. O. S.75. An der Genauigkeit der Angaben von Timonis zweifelte bereits 1755 der englische Gesandte in Konstantinopel, James Porter, der beauftragt war, über die Inokulationsmethode zu berichten. Er schrieb: «Timonis' account is incorrect, his facts are not to be depended on. Pylarinos is more accurate. » Dr. Maty, Queries sent to a Friend in Constantinople. In: Philosophical Transactions XLIX (1755), S.104.

27 Jenner, Edward, An Inquiry into the Causes and Effects of the Variolae Vaccinae, a disease discovered in some of the western counties of England, particularly Gloucestershire, and known by the name of the Cow Pox. Printed, for the author, by Sampson Low, Soho. London 1798.

${ }^{28}$ Fischer-Homberger, E., Geschichte der Medizin, Springer-Verlag, Berlin/Heidelberg/ New York 1975, S.167; 2.Aufl. S.170.

${ }^{29}$ Ein Kapuzinermönch, der während 16 Jahren in Georgien gelebt hatte, wußte nichts davon. Gentlemen's Magazine 25 (1755), S.452f. Zitiert bei G. Miller.

${ }^{30}$ Ropes, A. R., Lady Mary Wortley Montagu. Selected passages from her letters. Ch. Scribness, New York.

31 Ulrici, J.H., und Stoll, C.H., Methodus nova transplantandi variolas per insitionem. Das Blatter-Belzen, Wittenberg 1720.

32 Portmann, M.-L., Die Variolation im Spiegel der Korrespondenz Albrecht von Hallers (1708-1777) mit Achilles Mieg (1731-1799). In: Gesnerus 34 (1977), S. 294-303.

${ }^{33}$ Koelbing, H.M., Im Kampf gegen Pocken, Tollwut, Syphilis, Reihe Gute Schriften, Basel 1974.

34 Der französische Kliniker Armand Trousseau (1801-1867) wandte die Inokulation noch dann an, wenn ihm der Kuhpocken-Impfstoff ausgegangen war. Clinique médicale de l'Hôtel Dieu de Paris, Bd. 2, 1861. Zitiert bei M.-L.Portmann a.a. O. S. 302, Anm. 37. 


\section{Summary}

This paper deals with a predecessor of Jenner in the fight against smallpox. Inoculation or variolation was known in the southeastern countries (Greece, Turkey, Armenia, Georgia, the Caucasus Mountains, Persia). It was, however, only a lay treatment. Iakovos Pylarinos (1659-1718) came from the Ionian island of Cephalonia. He studied medicine at Padua. In 1701, the population of Constantinople was afflicted by a smallpox epidemic. Pylarinos, who lived there, became acquainted with a woman from Thessalia who inoculated many people. It was the first time that a physician adopted the technique. Pylarinos took the fluid from the pustule of a sick person. He made incisions into the arm or the thigh of the person to be inoculated and administered a few drops of the fluid into the incisions. Although he did this countless times, he never observed a fatal case. After he was convinced that the method was valid, he wrote an account of it, which was published in Venice in 1715. A summary appeared in the Philosophical Transactions, London, in 1716. The book was reprinted in Nuremberg (1717) and in Leiden (1718). A colleague of Pylarinos, Emmanuel Timonis, described the method of inoculation in a letter to John Woodward (Constantinople, December 1713); this letter was already printed in the Philosophical Transactions of 1714. Also Lady Mary Wortley Montagu wrote about the inoculation of smallpox in a famous letter (Adrianople, April 1717). This method was made known in England by Timonis, Pylarinos and Lady Mary. It was not before 1798 that the vaccination, which was propagated by Edward Jenner, could begin to succeed to the hitherto existing practice of variolation.

Dr. med. Stephanos Geroulanos

Universitätsspital Zürich

Chirurgische Universitätsklinik A

Rämistraße 100

8091 Zürich 